\title{
UMA PROPOSTA METODOLÓGICA DE ADEQUAÇÃO DE ÉPOCAS DE CULTIVO DESENVOLVIDA NA REGIÃO DE MANAUS - AM, ATRAVÉS DE BALANÇO HÍDRICO SERIADO'
}

\author{
Valéria Sucena HAMMES ${ }^{2}$, Nilson Augusto VILLA NOVA ${ }^{3}$
}

\begin{abstract}
RESUMO - O presente trabalho propõe uma metodologia para estabelecer os periodos adequados ao desenvolvimento vegetativo, trafegabilidade de máquinas e condições de colheita na região de Manaus - Amazonas, Brasil, a partir de balanço hídrico decendial de Thornthwaite \& Mather (1955). Os niveis de 30, 50, 70 e $100 \mathrm{~mm}$ de capacidade de água disponivel do solo foram arbitrados em função das características físico-químicas dos solos predominantes da região. De acordo com os resultados obtidos com o balanço seriado foram definidos critérios com três níveis de aptidão A (apto), R (restrito) e I (inapto) para cada parâmetro de avaliação. A consistência da metodologia é constatada pela concordância do modelo de adequação de cultivo às experimentações locais de otimização de época de plantio.
\end{abstract}

Palavras-chave: época de plantio, aptidão agrícola do solo, Manaus, Amazonia

Methodology for Selecting the Appropriated Cropping Time in the Region of Manaus-AM, Brazil, by the Climatological Water Balance

ABSTRACT - This work proposes a methodology to select the appropriated time for crop development, traffic of machines, and harvesting conditions in the region of Manaus, AM, Brazil, based on the Thornthwaite-Mather's climatological water balance. Soil water capacity were taken as $30,50,70$ and $100 \mathrm{~mm}$ as a function of the physical-chemical conditions of the prevailing soils in the regions. According to the results obtained from the water balance criteria were set up to define the level of appropriateness (Apt, Restrict or Inapt) for each evaluation parameter. Consistency of the methodology was checked against local field experimental results.

Key-words: crooping time, harvesting conditions, Manaus, Amazonia

\section{INTRODUÇÃO}

$\mathrm{Na}$ região equatorial, assim como na maior parte dos trópicos, a disponibilidade térmica não é fator limitante para a agricultura. Em Manaus, Amazonas, a temperatura média entre $24^{\circ}$ e $26^{\circ} \mathrm{C}$, com oscilação mensal que não ultrapassa a $1^{\circ} \mathrm{C}$, não determina limitação para culturas tropicais. Em contrapartida, a precipitação pluviométrica é uma restrição agrícola, seja por excesso de água (impedindo o cultivo do solo, prejudicando a maturação, impedindo a colheita, determinando condições favoráveis para moléstias, etc), ou seja, por déficit hídrico. Embora seja alta a precipitação pluviométrica ao longo do ano, a baixa profundidade efetiva do solo relacionada a toxidez do alumínio e alta taxa de evaporação causam "déficits hídricos" capazes de reduzir a produtividade dos cultivos

'Dissertação apresentada em agosto de 1994 à Escola Superior de Agricultura "Luiz de Queiroz", da Universidade de São Paulo, para obtenção do título de Mestre em Agronomia, na área de concentração de Agrometeorologia.

${ }^{2}$ Dra., Pesquisadora III. Laboratório de Diagnóstico e Gestão Ambiental, EMBRAPA Meio Ambiente. Caixa Postal 69, 13820-000, Jaguariuna, SP. E-mail: valeria@cnpma.embrapa.br. Autora

${ }^{3}$ Dr., Professor Associado. Departamento de Fisica e Meteorologia, ESALQ/USP. Caixa Postal 9, 13418-970, Piracicaba, SP. Bolsista do CNPq. Orientador 
agrícolas na região.

No processo de expansão da fronteira agrícola, através de projetos de ocupação induzida, com o propósito de ocupar os vazios demográficos, é comum a inexistência de uma tecnologia apropriada. Portanto torna-se necessário algum estudo preliminar para adequar espécies econômicas, cujos experimentos de campo exigem muitos anos de dedicação. Existe ainda, a polêmica sobre a preservação ecológica, que "esbarra" na necessidade de expansão de áreas produtivas com técnicas adequadas à exploração racional dos ecossistemas. Dai, visando a sustentabilidade desses sistemas agroflorestais pretende-se contribuir na busca de alternativas de produção com utilização mínima de insumos químicos (calcáreo, por exemplo) praticamente indisponiveis ou inviáveis economicamente nos recantos mais distantes do pais, como na Amazônia.

Nesta proposta metodológica, o cálculo da distribuição dos déficits através de um balanço hídrico sequencial para diferentes níveis de armazenamento, obtendo-se as limitações hídricas decorrentes para vários tipos de cobertura, tem por finalidade auxiliar não só os estudos agronômicos, mas também contribuir para a compreensão do comportamento dos ecossistemas nativos.

\section{MATERIAL E MÉTODO}

A base de dados climatológicos constituiu-se de uma série de 30 anos consecutivos de temperatura média e precipitação pluviométrica diários, do período de 1961 - 1990, da Estação localizada a $03^{\circ} 08^{\prime}$ 'S latitude $60^{\circ} 01^{\prime}$ 'W longitude e $72 \mathrm{~m}$ de altitude, em Manaus, no Estado do Amazonas. A caracterização físico-química dos solos predominantes na terra firme, que ocorre imediatamente ao norte e ao sul da planície inundável foi obtida pelas determinações de Corrêa (1984). Os cálculos do balanço hídrico Thohornthwaite \& Mather (1955) foram realizados de acordo com o programa de processamento elaborado por Barbieri et al. (1991), para intervalos de tempo decendiais. $\mathrm{O}$ programa foi utilizado para o cálculo dos armazenamentos em classe para cada decêndio (equação 1). $\mathrm{O}$ critério de EXC\% foi definido pela equação (2), e constitui-se simplesmente na \% na qual a chuva superou a capacidade de evapotranspiração, podendo existir mesmo quando ocorrem armazenamentos abaixo da CAD, expressando melhor a condição de molhamento do solo, importante para poder avaliar-se as condições de colheita, secagem natural e outras.

$$
\begin{aligned}
& \mathrm{CAD}_{\%}=(\mathrm{ARM} / \mathrm{CAD}) \times 100(1) \\
& \mathrm{EXC}_{\%}=\left(\mathrm{P}-\mathrm{ET}_{0} / \mathrm{ET}_{0}\right) \times 100(2)
\end{aligned}
$$

Considerou-se que a capacidade de armazenamento dos solos sob cobertura natural oscila em torno de 70 $\mathrm{mm} / \mathrm{metro}$. A determinação dos niveis de CAD arbitrados apresentados na tabela 1 seguiu a recomendação de Reichardt et al. (1980) para diferentes profundidades de exploração radicular, com espécies cultivadas anuais e perenes. 
Tabela 1: Niveis de CAD correspondentes às profundidades aproximadas

\begin{tabular}{cc}
\hline CAD $(\mathrm{mm})$ & Profundidade $(\mathrm{cm})$ \\
\hline 30 & 40 \\
50 & 70 \\
70 & 100 \\
100 & 150 \\
\hline
\end{tabular}

Adotou-se metodologia similar àquela desenvolvida por Tommaselli (1992) para análise das melhores épocas de plantio. Em função da frequência relativa (tempo de retorno), estipulou-se os seguintes critérios de avaliação para armazenamento, trafegabilidade e colheita.

De acordo com Doorembos \& Kassan (1979) para um valor de evapotranspiração, situado entre 4 e 5 $\mathrm{mm} / \mathrm{dia}$, a maior parte das plantas fica sob "stress" hídrico quando o nível de armazenamento é $<50 \%$ da CAD. Os niveis de aptidão para armazenamento crítico estão apresentados na Tabela 2 .

Tabela 2: Niveis de risco para déficit de armazenamento $\leq 50 \%$ da CAD

\begin{tabular}{cc}
\hline Classes de risco & Frequência relativa \\
\hline Apto (A) & $\leq 20 \%$ \\
Restrito (R) & $>20 \% \leq 40 \%$ \\
Impróprio (I) & $>40 \%$ \\
\hline
\end{tabular}

Segundo Bolton et al. (1968) a condição de aptidão para trafegabilidade para solo argiloso é $\mathrm{CAD} \% \leq 70 \%$, sendo que os níveis de risco arbitrados estão apresentados na Tabela 3.

Tabela 3. Niveis de risco para trafegabilidade para $\mathrm{CAD} \% \leq 70 \%$

\begin{tabular}{cc}
\hline Classes de risco & Frequência relativa \\
\hline Apto (A) & $\geq 80 \%$ \\
Restrito (R) & $<80 \mathrm{e} \geq 60 \%$ \\
Impróprio (I) & $<60 \%$ \\
\hline
\end{tabular}

Adotou-se como nível crítico para colheita e secagem natural a situação na qual o excesso hídrico do decêndio (EXC\%) seja nulo. Os niveis de aptidão arbitrados estão apresentados na Tabela 4.

Tabela 4. Niveis de aptidão para colheita e secagem natural para probabilidade de ocorrência $\geq 70 \%$

\begin{tabular}{cc}
\hline Classes de risco & Frequência relativa \\
\hline Apto (A) & $\geq 70 \%$ \\
Restrito (R) & $\leq 70$ e $\geq 60 \%$ \\
Impróprio (I) & $<60 \%$ \\
\hline
\end{tabular}

Devido ao fato da distribuição de frequência relativa, tanto dos déficits como excessos, mostrarem um elevado grau de descontinuidade, não foi possível estabelecer as funções de frequência acumulada $x$ classes. As frequências relativas foram calculadas então, pelo número de eventos $\geq$ que a classe considerada. 


\section{RESULTADOS}

\section{Classificação climática}

As variações dos elementos meteorológicos segundo uma regularidade diferencial de intensidade e distribuição condicionam a existência de climas. Wilhelm Köeppen estabeleceu uma classificação baseada no estudo da vegetação, associando valores numéricos de temperatura e precipitação a essas regiões. W.C.Thornthwaite utilizou a temperatura, a precipitação pluviométrica e a evapotranspiração potencial como elementos de classificação climática.

Segundo a classificação de Köeppen, a região de Manaus caracteriza-se pelo clima $A_{f}$ e $A_{w}$ Subentende-se, entre outras coisas, que o total de precipitação anual elevado minimiza os efeitos do período de estiagem. Também Thorthwaite caracteriza o clima da região de Manaus - se como superúmido com pequena ou nenhuma deficiência de água, megatérmico com vegetação durante o ano todo. Tais definições não se enquadram às condições agronômicas reais da região, induzindo o técnico a erro, pois conforme os resultados da avaliação de aptidão agrícola do solo, verificouse que a existência de severos déficits hídricos ao longo do ano vão restringir as épocas de cultivo para várias culturas. Portanto, sugere-se a revisão das classificações climáticas, como elemento de informação primária a qualquer pesquisa agronômica local.

\section{Aptidão agrícola do solo}

No presente trabalho com o estudo probabilístico da distribuição destes déficits procurou-se estabelecer os níveis de risco para cada época de plantio em função do tempo de retorno destes déficits, assim como niveis de risco de excesso hídrico, para quatro classes de capacidade de água disponivel. A tabela 5 possibilita uma visão conjunta das condições de aptidão Apta (A), Restrita (R) e Inapta(I) para ciclo vegetativo, trafegabilidade e colheita, segundo os critérios de nível de riscos pré-estabelecidos e o cálculo das frequências relativas percentuais obtidos a partir do balanço hídrico decendial.

\section{DISCUSSÃO}

Pode-se observar que o início das épocas de aptidão por déficit hidrico ocorrem praticamente na $36^{\mathrm{a}}$ década do ano, correspondente ao $3^{\circ}$ decêndio de dezembro, para todas as CADs. Ao considerar-se o nivel de $80 \%$ de probabilidade do evento, determina-se um atraso no periodo de cultivo (pois deveria iniciar-se em outubro) e um minimo de risco até para a CAD de $30 \mathrm{~mm}$, condicionados pela variabilidade das precipitações detectada pelo balanço hidrico seriado. Do contrário, as ocorrências consideradas restritas poderiam ser consideradas aptas e expor $\mathrm{o}$ agricultor ao risco de perdas de até $40 \%$. Assim, também foi influenciado o período final da época de aptidão, que se estendeu de maio até junho, de acordo 
Tabela 5. Classificação dos niveis de aptidào agricola do solo de terra-firme de Manaus, AM

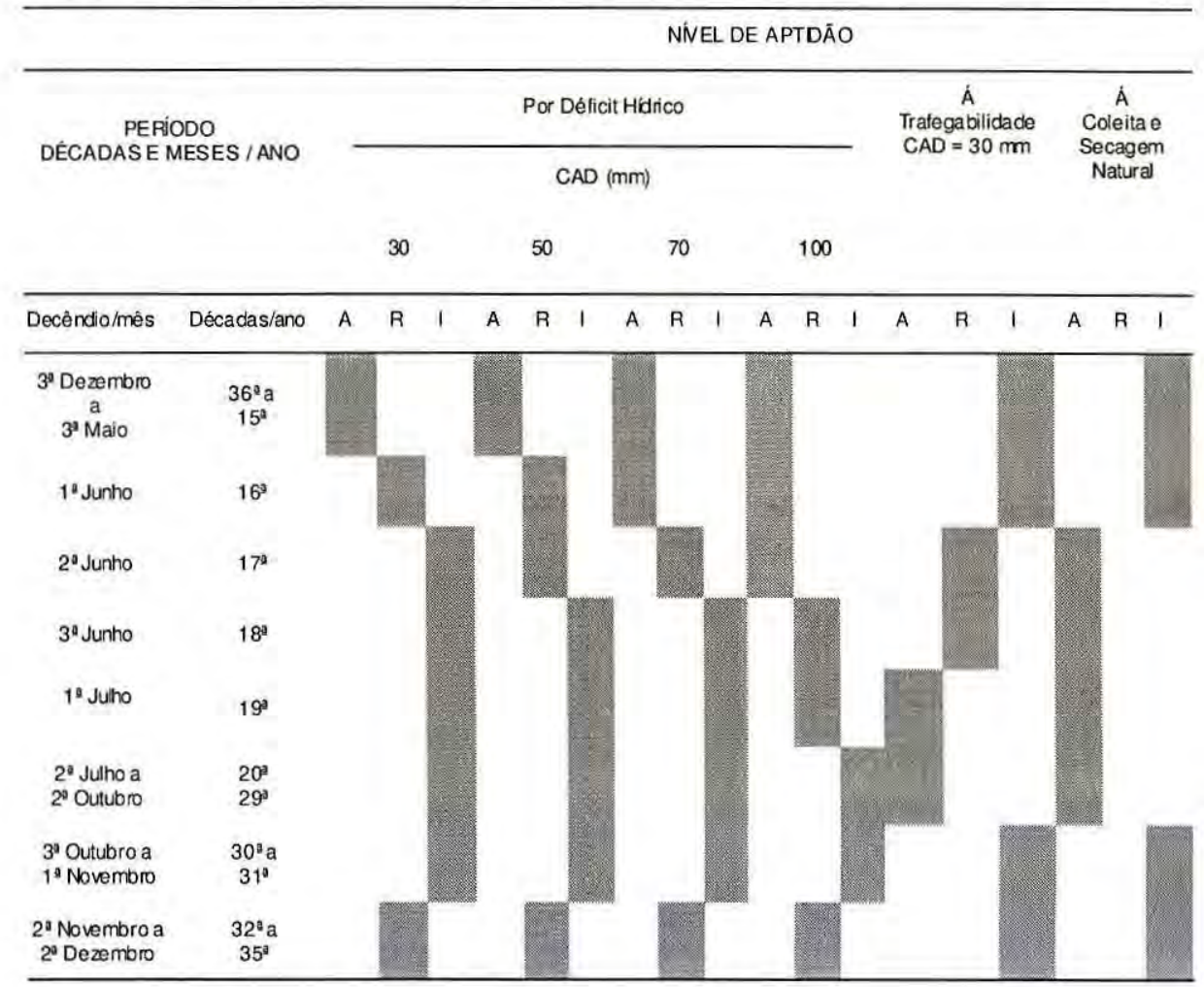

com o valor crescente da CAD considerada e o decréscimo dos niveis de inaptidão por déficit hídrico (I).

É óbvio, que os limites dos niveis de aptidão a trafegabilidade (condição adequada do solo ao tráfego de máquinas agrícolas motorizadas convencionais usadas no preparo do solo e colheita) são maiores quando as máquinas são mais leves e as operações são mais simples, assim como em condição de solo arenoso, cuja drenagem é mais rápida. Contudo, só se dispõe de 3 meses e meio (de julho a meados de outubro) com condições do solo para ser trabalhado mecanicamente.
Ainda avaliando o tabela 5, todas as culturas que necessitam de restrição de água na fase de maturação, seja por problemas fitossanitários ou de secagem natural, deverão cumprir o período de colheita, com inicio em fins de junho até meados de outubro, período no qual há $70 \%$ de probabilidade com precipitações sempre inferiores ou no máximo iguais ao valor da evapotranspiração (vide o conceito de EXC\%).

Pode-se concluir que na região, o preparo mecanizado do solo está restrito ao periodo de junho a início de dezembro, o plantio deverá efetivar-se a partir do final de dezembro até o fim 
do mês de maio, dependendo do ciclo, prevendo-se que a fase de maturação e colheita, iniciem no mínimo no mês de junho.

Depois de conduzir experimentos sobre sistema de produção do feijão-caupi (Vigna unguiculata (L.) Walp), a UEPAEEMBRAPA - Manaus recomenda através da circular técnica $\mathrm{n}^{\circ} 4$ que a semeadura em terra firme seja realizada na segunda quinzena de abril em zona de solos arenosos (CAD próxima de $30 \mathrm{~mm}$ ), em solos mais argilosos (CAD próxima a $50 \mathrm{~mm}$ ) o plantio poderá atrasar-se até a $2^{a}$ quinzena de maio. Tal condição está plenamente de acordo com as condições fornecidas pelo modelo. Verificou-se ainda concordância entre a observação e os critérios propostos resultantes dos experimentos conduzidos (1982 a 1985) por Galvão et al. (1987) para definição das épocas de plantio de arroz (Oryza sativa L. variedade IAC-17), soja (Glicyne max (L) Merril - variedade Tropical e Jupiter), milho (Zea mays, L. variedades BR5102 ou BR5110) e feijão-caupi ((Vigna unguiculata (L.) Walp - variedades Manaus, VITA3 e IPEAN V-69). De acordo com o modelo, para cultura de ciclo em torno de 4 meses, o preparo do solo em terra firme pode ser feito de julho a meados de outubro e o plantio em março. Yuyama (1979) confirma o mês de março como a melhor época de plantio para cultivares de soja, milho e outras.

Acredita-se que os problemas gerais de fitossanidade na região (flutuação de populações de pragas, potencial de infestaçâo de moléstias) estão intimamente relacionadas ao balanço hídrico do solo, devido ao regime térmico praticamente constante. Como exemplo da integração clima $\mathrm{x}$ doenças limitadoras podemos citar Coltri (1989), que após experimento, indicou restrições de cultivo da cenoura na região, apontando o início de maio para plantio, quando a água no solo é favorável e conduz à fase de formação da produção para o periodo mais seco, escapando da queima das folhas e podridão da raiz, doenças estas que ocorrem com maior frequência em periodos de elevada precipitação como demonstra o modelo.

A definição da área de abrangência do modelo proposto pode ser obtida ao efetuar-se os mesmos cálculos para diferentes áreas próximas e compará-las. Sabendo-se ainda que a maior parte das chuvas na região é de origem convectiva e elevada variação espacial e como não se dispunha de dados com série tão longas, realizou-se apenas o confronto das condições de armazenamento ao longo de 5 anos, com informações obtidas de cinco estações experimentais próximas, que possibilitou perceber alguma similaridade no comportamento dos armazenamentos. Assim sendo a extensão de validez dos cálculos

\section{CONCLUSÃO}

Em face dos resultados do modelo de aptidão agrícola do solo serem compativeis com aqueles 
indicados pela experimentação na região, conclui-se que a metodologia de adequação de épocas de plantio proposta é consistente e permite a racionalização de futuros experimentos.

\section{Bibliografia citada}

Barbieri, W.; Tuon, R.L.; Angelocci, L.R. 1991. Programa de balanço hidrico (Thornthwaite \& Mather, 1955). Anais Congresso Brasileiro de Agrometeorologia, 7. Viçosa. p.297-301.

Bolton, B. 1968. Days suitable for fieldwork Mississipi River Delta cotton area. Louisiania. Research Report, 368. Louisianis State University/Agricultural Experiment Station.

Coltri, M.L. 1989. Produção de cenoura na região de Manaus, Amazonas. Manaus, EMBRAPA/UEPAE Manaus. Circular Técnica. $15 \mathrm{p}$.

Corrêa, J.C. 1984. Caracteristicas físicohidricas dos solos Latossolo amarelo, Podzólico vermelho-amarelo e Podzo Hidromórfico do Estado do Amazonas. Pesquisa Agropecuária Brasileira, Brasilia, 19(3): 347-360

Doorembos, J.; Kassan, A.H. 1979. Yield response to water. Roma, FAO. Irrigation and Drainage Paper, 33. 193p.

Galvão, E.U.; Barreto, J.F.; Corrêa, J.C.; Cravo, M.; Dias, M. 1987. Planting dates in relation to weather pattern at Manaus, Brazil. North Carolina State University. Tropsoil technical report 1985. Raleigh. p.107-108.
Nogueira, O.L. 1981. Cultura de feijão caupi no Estado do Amazonas. Manaus, EMBRAPA/UEPAE Manaus. Circular Técnica, 4: 21p.

Reichardt, K.; Ranzani, G.; Freitas Jr., E.; Libardi, P.L. 1980. Aspectos hídricos de alguns solos da Amazônia - Região do baixo Riuo Negro. Acta Amazonica, 10 (1): $43-46$

Thornthwaite, C.W.; Mather, J.R. 1955. The water budget and its use in irrigation. Estados Unidos. Department of Agriculture. The Water Balance. Washington, p. 346-357.

Tommaselli, J.T.G. 1992. Deficièncias hidricas no solo e épocas de plantio de milho (Zea mays) em Cambará e Londrina - PR. Piracicaba, SP. Tese de mestrado ESALQ/SP. 78 p.

Yuyama, K. 1979. Como cultivar soja na Amazônia. In: Pahlen, A. von der; Kerr, W. E.; Paiva, W. O; Rahman, F.; Yuyama, K.; Noda, H. 1979. Introdução a horticultura e fruticultura no Amazonas. INPA, Manaus: 124-130. 